\title{
Performance of a risk score for predicting preterm pre-eclampsia. (Mini-commentary on BJOG-19-0970.R4)
}

\author{
Ulla Sovio ${ }^{1}$ \\ ${ }^{1}$ University of Cambridge
}

April 28, 2020

Mini-commentary on BJOG-19-0970.R4: External validation of a simple risk score based on the ASPRE trial algorithm for preterm preeclampsia considering maternal characteristics in nulliparous pregnant women: a multicentric retrospective cohort study

\section{Performance of a risk score for predicting preterm pre-eclampsia}

Ulla Sovio

Cambridge University

The Rosie Hospital

Robinson Way

Cambridge

Email: us253@medschl.cam.ac.uk

Brunelli et al (BJOG 2020) evaluated a previously validated maternal history -based risk score in a multicentric retrospective cohort from Italy. The score discriminated poorly in the Italian cohort (AUC $=0.659[95 \%$ CI 0.579-0.726]) but it discriminated well in the Pregnancy Outcome Prediction (POP) study (AUC=0.846 [95\% CI 0.787-0.906]) (Sovio et al, BJOG 2019;126:963-970), although the proportion of women who developed preterm pre-eclampsia was similar in both studies ( $1.0 \%$ and $0.7 \%$, respectively). I will discuss possible reasons for the difference and the next steps for evaluating the score.

The prevalence of the maternal risk factors that constitute the risk score was very low in the Italian study. Chronic hypertension has a major contribution, i.e. it strongly predicts (superimposed) preterm pre-eclampsia. Only $0.5 \%$ of all the women and $15 \%$ of the cases had chronic hypertension, whereas in the POP study, the prevalences were 5\% and 35\%, respectively (Sovio et al, Hypertension 2017;69(4):731-738). An analysis of the SCOPE study (Myers, Pregnancy Hypertension 2019;17:S21) illustrated that the performance of the risk score was similarly poor in a healthy nulliparous population which excluded all women with chronic hypertension ( $\mathrm{AUC}=0.661$ [95\% CI 0.596-0.725]). This is hardly surprising.

The representativeness of the cohort was not clear from the article. A comparison between recruited women and eligible non-recruited women would help in assessing selection bias. Misclassification may also contribute to the poor performance. In the POP study, pre-eclampsia was ascertained through a careful review of case records and linkage to electronic databases (Sovio et al, 2017). The quality of the electronic data used as a basis of defining pre-eclampsia in the Italian study was not discussed in the article. Both ISSHP and ACOG define cut-offs using "[?]" (hypertension: [?]140/[?]90 $\mathrm{mmHg}$, proteinuria: [?]300mg/day), whereas the Italian study used ">". Resulting misclassification may be minor, but this depends on the extent of zero end-digit preference. Moreover, measurement error or misreporting of risk factors or aspirin use could have contributed to an increased random error or bias in the risk score. 
I suggest that future evaluations of the risk score should be performed in datasets representative of the underlying population in terms of prior maternal risk. Only the women undergoing interventions which may alter the risk of preterm preeclampsia should be excluded. The risk score has already been validated against the model for predicted gestational age at preeclampsia (PGAPE) in nulliparous women (Sovio et al, 2019; Myers, 2019). Brunelli et al (2020) did not attempt to validate the risk score against PGAPE but instead compared it with the full FMF algorithm, and the latter performed better. The need for additional measurements to those included in the risk score was expected, given the low prevalence of prior maternal risk factors (Myers, 2019).

A suggested scoring for maternal risk has been published for parous women (Sovio et al, 2019). Evaluation of the risk score and validation against PGAPE in parous women is now needed since this could not be done in the POP study or in the SCOPE study.

Disclosures: Dr. Sovio reports grants from NIHR Cambridge Biomedical Research Centre, during the conduct of the study. In addition, Dr. Sovio has a patent application for a novel predictive test for fetal growth restriction (FGR) pending. A completed disclosure of interest form is available to view online as supporting information. 